\title{
Radio electric asymmetric brain stimulation in the treatment of behavioral and psychiatric symptoms in Alzheimer disease
}

This article was published in the following Dove Press journal:

Clinical Interventions in Aging

25 July $201 \mathrm{I}$

Number of times this article has been viewed

\author{
Piero Mannu' \\ Salvatore Rinaldi ${ }^{1,2}$ \\ Vania Fontani' \\ Alessandro Castagna' \\ 'Rinaldi Fontani Institute, Department \\ of Neuro Psycho Physio Pathology, \\ Florence, Italy; ${ }^{2}$ Medical School of \\ Occupational Medicine, University \\ of Florence, Florence, Italy
}

Purpose: Behavioral and psychiatric symptoms of dementia (BPSD) are common in Alzheimer's disease $(\mathrm{AD})$ and disrupt the effective management of $\mathrm{AD}$ patients. The present study explores the use of radio electric asymmetric brain stimulation (REAC) in patients who have had a poor response to pharmacological treatment.

Patients and methods: Eight patients (five females and three males; mean [ \pm standard deviation] age at study baseline: $69.9 \pm 3.0$ years) diagnosed with AD according to the DSM-IV-TR criteria (mean onset age of $\mathrm{AD}$ : $65.4 \pm 3.5$ years) were cognitively and psychometrically assessed with the Mini-Mental State Examination (MMSE), the Activity of Daily Living (ADL), the Instrumental Activity of Daily Living (IADL), and the Neuropsychiatric Inventory (NPI), prior to and after each of 2 REAC treatment cycles.

Results: Scores on the MMSE and all subscales of the NPI (frequency, severity, and distress), the ADL, and the IADL were significantly improved following the initial REAC treatment. There was further significant improvement in all measurements (with a tendency for improvement in the IADL) after the second REAC treatment cycle.

Conclusion: The improvement of cognitive and behavioral/psychiatric functioning following REAC treatment suggests that this innovative approach may be an effective, safe, and tolerable alternative to pharmacological treatment of AD patients, especially in the area of BPSD. Elderly patients suffering from other types of dementia may also benefit from REAC treatment.

Keywords: anxiety, depression, insomnia, behavioral and psychiatric symptoms of dementia (BPSD)

\section{Introduction}

The progressively increasing number of the elderly in the general population and, consequently, the growing prevalence of Alzheimer disease ${ }^{1,2}$ (AD) highlight the need for new specifically targeted therapeutic options. ${ }^{3-6}$ The currently available psychological ${ }^{7-10}$ and psychopharmacological ${ }^{11-15}$ treatments $^{16}$ for AD provide relatively poor results. The inadequate and/or insufficient safety and tolerability profile of 'typical' AD therapies produce familiar social and economic difficulties. ${ }^{17,18}$ According to the Diagnostic and Statistical Manual of Mental Disorders, Fourth Edition, Text Revision (DSM-IV-TR), AD includes a disruption in attention, ${ }^{19,20}$ concentration, memory, ${ }^{21,22}$ and cognition. ${ }^{23}$ While the DSM-IV-TR instructs diagnosing clinicians to specify whether cognitive symptoms of AD are accompanied by behavioral abnormalities (eg, delusions, depression, anxiety, agitation, wandering, sexual behavior alterations, sleep dysfunctions, disorientation), behavioral symptoms of AD are not included in the working definition.
Correspondence: Salvatore Rinaldi Rinaldi Fontani Institute,

Viale Belfiore 43,

50144 Florence, Italy

Tel +39055 290307

Fax +39055 290399

Email srinaldi@irf.it 
Table I Total score variation before and after first REAC treatment cycle

\begin{tabular}{|c|c|c|c|c|c|}
\hline Test & $\begin{array}{l}\text { TSV before } \\
\text { REAC }\end{array}$ & $\begin{array}{l}\text { TSV after } \\
\text { I REAC cycle }\end{array}$ & $\begin{array}{l}\text { ANOVA } \\
F\end{array}$ & $d f$ & $P$ \\
\hline MMSE & $18.5 \pm 2.4$ & $23.4 \pm 2.3$ & 64.0588 & 14 & $<0.01$ \\
\hline NPI for "frequency" & $32.2 \pm 2.5$ & $26.4 \pm 2.4$ & 309.8421 & 14 & 0.000 \\
\hline NPI for "severity" & $28.6 \pm 3.4$ & $22.1 \pm 3.4$ & 529.000 & 14 & 0.000 \\
\hline NPI for "distress" & $49.5 \pm 3.6$ & $41.0 \pm 2.1$ & 583.1538 & 14 & 0.000 \\
\hline$A D L$ & $3.8 \pm 1.0$ & $4.9 \pm 0.5$ & 33.8507 & 14 & NS \\
\hline IADL & $10.0 \pm 2.4$ & $13.0 \pm 1.1$ & |34.8667 & 14 & 0.000 \\
\hline
\end{tabular}

Abbreviations: REAC, radio electric asymmetric brain stimulation; TSV, total score variation; MMSE, Mini-Mental State Examination; NPI, Neuropsychiatric inventory; ADL, Activity of Daily Living; IADL, Instrumental Activity of Daily Living; NS, not statistically significant.

The relationship between $\mathrm{AD}$ and the behavioral and psychiatric symptoms of dementia ${ }^{24}$ (BPSD) is becoming more prevalent. Symptoms of dementia accompany AD in about $90 \%$ of cases ${ }^{25}$ typically arising early in the course of the disease and persisting. Unlike the steady loss of "global" cognition, throughout the course of AD, behavioral symptoms are more variable, with different types of BPSD seen among patients. ${ }^{26}$ Despite the observed inter-patient variability in number and type of behavioral symptoms encountered in $\mathrm{AD}$, patients with advanced illness tend to have more behavioral symptoms than those in earlier phases of the disorder. Several long-term studies indicate that once a specific symptom occurs in a given patient, it is likely to persist or recur thereafter.

Radio electric asymmetric brain stimulation ${ }^{27,28}$ (REAC) treatment has proven efficacy in ameliorating several stress-related disorders, depression, and anxiety, ${ }^{29-35}$ and our unpublished data have shown promising results for some forms of dementias. REAC treatments are painless, noninvasive, and have a high safety and tolerability profile. On the basis these we can hypothesize that the treatments with REAC technology may be helpful in AD and, in general, in several forms of cognitive-impairment disorders.

\section{Aim of the present study}

REAC has demonstrated efficacy in improving certain psychiatric disorders such as anxiety, depression and bipolar disorder. The main goal of the present study was to investigate the efficacy of REAC in the treatment of behavioral symptoms in $\mathrm{AD}$ patients. In addition, effects on cognitive functioning were also investigated.

\section{Materials and methods}

The data for the current study were collected during routine therapy sessions at the Rinaldi Fontani Institute, Behavioral Disorder Department in Florence, Italy. Most patients referred to the clinic were nonresponders to typical pharmacological strategies. Eight patients (five females and three males; mean $[ \pm \mathrm{SD}]$ age at study baseline: $69.9 \pm 3.0$ years) diagnosed with AD according to the DSM-IV-TR criteria (mean onset age of AD: $65.4 \pm 3.5$ years), presenting with behavioral and/ or psychiatric disturbances were psychometrically assessed using the Mini-Mental State Examination ${ }^{36}$ (MMSE), the Activity of Daily Living ${ }^{37}$ (ADL), the Instrumental Activity of Daily Living ${ }^{38}$ (IADL), and the Neuropsychiatric Inventory $^{39}(\mathrm{NPI})$, before and after 2 treatment cycles of REAC.

REAC is applied by a medical device that is based on an innovative biostimulation technology. ${ }^{27,28}$ REAC works within a typical frequency range of $2.4,5.8$, or $10.5 \mathrm{GHz}$, selected by the operator for each specific protocol. For the current study, a frequency of $10.5 \mathrm{GHz}$, with a specific absorption rate (SAR) of $7 \mu \mathrm{W} / \mathrm{kg}$, was used. The neuro postural optimization (NPO) protocol consisting of a single radiofrequency burst of 500 milliseconds (ms) applied by touching the metallic tip of the REAC probe (Convogliatore di Radianza Modulante CRM; ASMED, Italy) to the ear pavilion was performed as an initial treatment. NPO was followed by another treatment protocol, named neuro psycho physical optimization (NPPO). This protocol consisting of seven REAC radiofrequency bursts of $500 \mathrm{~ms}$ applied by touching the metallic tip of

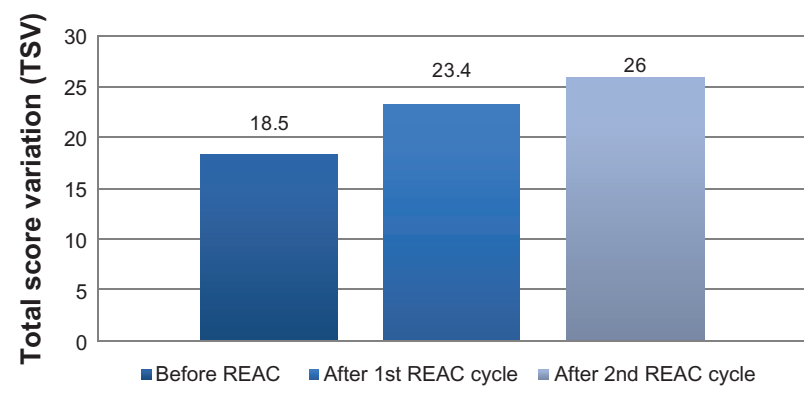

Figure I Mini-Mental State Examination (MMSE) before, after the first and second REAC treatment cycle.

Abbreviations: REAC, radio electric asymmetric brain stimulation; TSV, total score variation. 


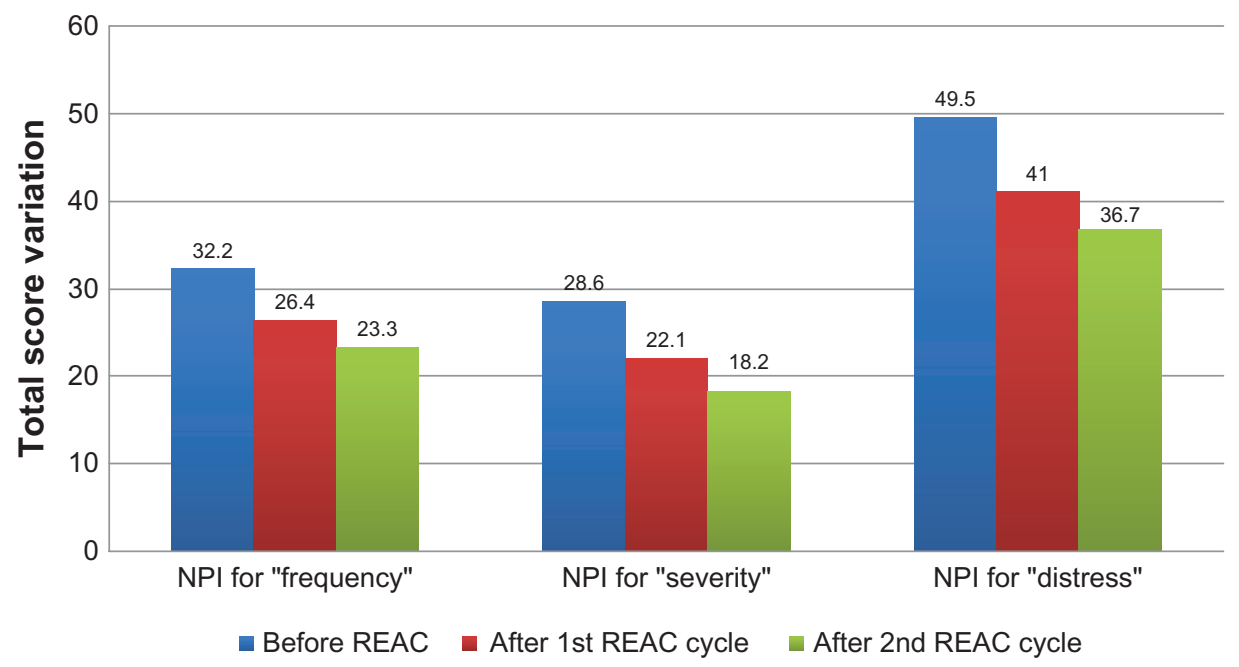

Figure 2 Neuro Psychiatric Inventory (NPI) before after first and second REAC treatment cycle. Abbreviation: REAC, radio electric asymmetric brain stimulation.

the REAC probe at precise points of the ear. Each therapy session lasts about 5 seconds. An NPPO treatment cycle consists of 18 therapy sessions, administered on alternate days. The mean interval between the first and the second NPPO treatment cycle is about 6 months.

ANOVA for repeated measures was performed for statistical analysis of differences in MMSE, ADL, IADL, and NPI scores prior to and following REAC treatments. $P$ values $<0.05$ were considered significant.

\section{Results}

After the first REAC treatment cycle, patients showed great improvement in cognitive performance as demonstrated by a significant increase in average MMSE total score from $18.5( \pm 2.4)$ to $23.4( \pm 2.3)$ (ANOVA $P<0.01)$ (Table 1 and Figure 1). In addition, BPSD was improved as demonstrated by a decrease in average NPI frequency score (Table 1 and Figure 2) from $32.2( \pm 2.5)$ to $26.4( \pm 2.4)$ (ANOVA $P=0.000$ ), NPI severity score (Table 1 and Figure 2 ) from

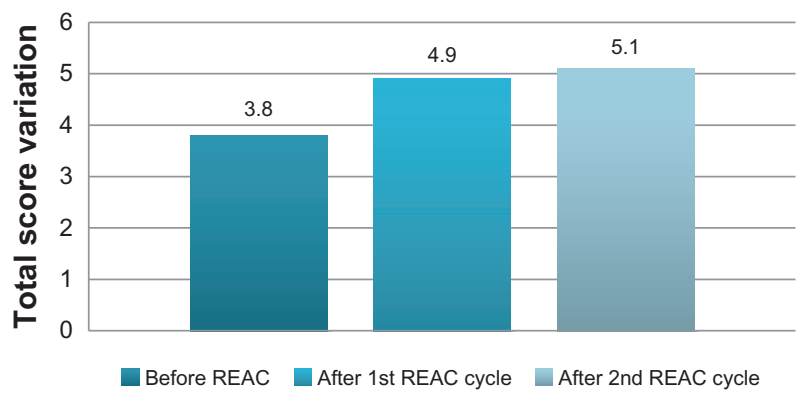

Figure 3 Activity of Daily Living (ADL), before, after first and second REAC treatment cycle.

Abbreviation: REAC, radio electric asymmetric brain stimulation.
$28.6( \pm 3.4)$ to $22.1( \pm 3.4)$ (ANOVA $P=0.000)$, and NPI distress score from (Table 1 and Figure 2) $49.5( \pm 3.6)$ to $41.0( \pm 2.1)$ (ANOVA $P=0.000)$. In addition, average ADL score (Table 1 and Figure 3$)$ increased from $3.8( \pm 1.0)$ to 4.9 $( \pm 0.5)$ (ANOVA $P=$ NS) and average IADL score (Table 1 and Figure 4) increased from $10.0( \pm 2.4)$ to $13.0( \pm 1.1)$ (ANOVA $P=0.000$ ).

After the second REAC treatment cycle, there were further significant ameliorations in both cognitive and behavioral performance (Table 2). There was a significant increase in average MMSE total score (Table 2 and Figure 1) to $26.0( \pm 3.4)$ (ANOVA $P<0.001$ ), and a significant decrease in NPI frequency score (Table 2 and Figure 2) to $23.3( \pm 2.3)$ (ANOVA $P<0.001$ ), NPI severity score (Table 2 and Figure 2$)$ to $18.2( \pm 3.7)$ (ANOVA $P=0.000$ ), and NPI distress score (Table 2 and Figure 2$)$ to $36.7( \pm 4.3)$ (ANOVA $P=0.000$ ). IADL total score was significantly increased after the second REAC treatment to $14.4( \pm 0.7)$ (ANOVA $P=$ NS) (Table 2 and Figure 4). The increase in

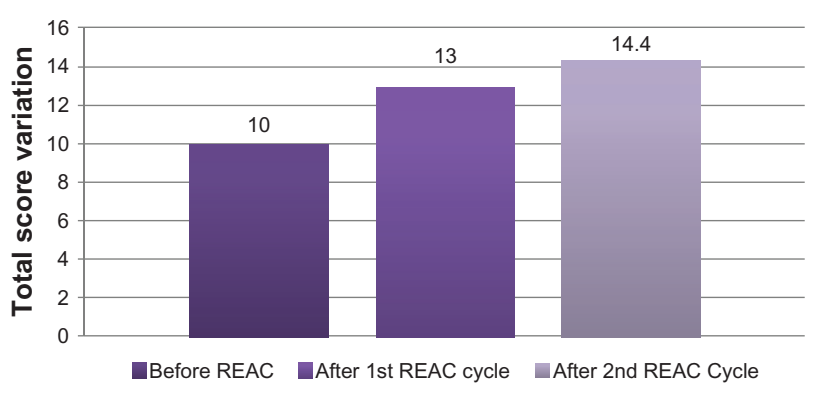

Figure 4 Instrumental Activity of Daily Living (IADL), before, after first and second REAC treatment cycle.

Abbreviation: REAC, radio electric asymmetric brain stimulation. 
Table 2 Total score value of first versus second REAC treatment cycle

\begin{tabular}{|c|c|c|c|c|c|}
\hline Test & $\begin{array}{l}\text { TSV after } \\
\text { I REAC cycle }\end{array}$ & $\begin{array}{l}\text { TSV after } \\
2 \text { REAC cycle }\end{array}$ & $\begin{array}{l}\text { ANOVA } \\
F\end{array}$ & $d f$ & $P$ \\
\hline MMSE & $23.4 \pm 2.3$ & $26.0 \pm 3.4$ & 44.800 & 14 & $<0.001$ \\
\hline NPI for "frequency" & $26.4 \pm 2.4$ & $23.3 \pm 2.3$ & 79.5455 & 14 & $<0.001$ \\
\hline NPI for "severity" & $22.1 \pm 3.4$ & $18.2 \pm 3.7$ & 203.8936 & 14 & 0.000 \\
\hline NPI for "distress" & $4 I .0 \pm 2.1$ & $36.7 \pm 4.3$ & 191.6526 & 14 & $<0.05$ \\
\hline$A D L$ & $4.9 \pm 0.5$ & $5.1 \pm 0.4$ & 0.4430 & 14 & NS \\
\hline IADL & $13.0 \pm 1.1$ & $14.4 \pm 0.7$ & 0.7238 & 14 & NS \\
\hline
\end{tabular}

Abbreviations: REAC, radio electric asymmetric brain stimulation; TSV, total score variation; MMSE, Mini-Mental State Examination; NPI, Neuropsychiatric inventory; ADL, Activity of Daily Living; IADL, Instrumental Activity of Daily Living; NS, not statistically significant.

ADL total score to $5.1 \pm 0.4$ (Table 2 and Figure 3 ) was not statistically significant (ANOVA $P=\mathrm{NS}$ ).

\section{Discussion}

REAC treatment enhanced cognitive and behavioral functioning in patients with AD. All measures of cognitive functioning were significantly improved after the initial REAC treatment and continued to improve after the second REAC cycle. Similarly, all behavioral measurements were positively affected after the first REAC treatment and most continued to improve after the second cycle.

It is likely that the effects on cognition and behavior are due to a process of synchronization of brain function caused by the microelectric stimulation of the REAC. ${ }^{29}$

While cognitive functioning benefitted from REAC treatment, the positive effects on BPSD may be of more importance in the management of AD patients. The improvement in these clinical parameters is reflected in functional terms by an increase in the quality of life of patients as reported by relatives, physicians, and caregivers and is psychometrically demonstrated by the ADL and IADL total scores. The use of REAC treatment may decrease the need to continually alter pharmacological treatments to achieve optimal results, leading to significant savings in economic resources of rehabilitation programs for $\mathrm{AD}$ patients.

REAC treatments alone are not likely to manage all deficits associated with neuropsychiatric disorders in the elderly. However, the results of the present study suggest that REAC treatment has a high safety, tolerability, and efficacy profile and may be useful not only in patients who have experienced poor and/or unstable responses to psychotropic drugs, but also as a first-line therapeutic option. REAC treatment may be useful not only for AD patients, but also for other forms of dementia (ie, vascular dementia or mixed conditions).

\section{Acknowledgments}

The authors thank Dr Eng Matteo Lotti Margotti for data analysis. Lucia Aravagli MD and Stefania Bini MD of Rinaldi Fontani Institute, Department of Neuro Psycho Physio Pathology, Florence, Italy, for their helpful discussions.

\section{Disclosure}

Salvatore Rinaldi and Vania Fontani are the inventors of the Radio Electric Asymmetric Conveyer.

\section{Reference}

1. Chouliaras L, Rutten BP, Kenis G, et al. Epigenetic regulation in the pathophysiology of Alzheimer's disease. Prog Neurobiol. 2010;90(4): 498-510.

2. Salloway S. Introduction: the prevalence of Alzheimer's disease - a growing crisis. CNS Spectr. 2008;13(3 Suppl 3):1.

3. Lleo A. Current therapeutic options for Alzheimer's disease. Curr Genomics. 2007;8(8):550-558.

4. Moreira PI, Zhu X, Nunomura A, Smith MA, Perry G. Therapeutic options in Alzheimer's disease. Expert Rev Neurother. 2006;6(6): 897-910.

5. Garcia-Ruiz Espiga PJ, Echeverria A, Garcia-Torres A, Contreras A. New therapeutic options in Alzheimer's disease. Rev Neurol. 2006; 42(8):478-481.

6. Kurz A, Perneczky R. Novel insights for the treatment of Alzheimer's disease. Prog Neuropsychopharmacol Biol Psychiatry. 2011;35(2): 373-379.

7. Ballard C, Corbett A, Chitramohan R, Aarsland D. Management of agitation and aggression associated with Alzheimer's disease: controversies and possible solutions. Curr Opin Psychiatry. 2009;22(6): 532-540.

8. Mathias JL, Burke J. Cognitive functioning in Alzheimer's and vascular dementia: a meta-analysis. Neuropsychology. 2009;23(4):411-423.

9. Logsdon RG, McCurry SM, Teri L. Evidence-based psychological treatments for disruptive behaviors in individuals with dementia. Psychol Aging. 2007;22(1):28-36.

10. O'Connor DW, Ames D, Gardner B, King M. Psychosocial treatments of psychological symptoms in dementia: a systematic review of reports meeting quality standards. Int Psychogeriatr. 2009;21(2): 241-251.

11. Rocca P, Marino F, Montemagni C, Perrone D, Bogetto F. Risperidone, olanzapine and quetiapine in the treatment of behavioral and psychological symptoms in patients with Alzheimer's disease: preliminary findings from a naturalistic, retrospective study. Psychiatry Clin Neurosci. 2007; 61(6):622-629. 
12. Lauterbach EC, Victoroff J, Coburn KL, Shillcutt SD, Doonan SM, Mendez MF. Psychopharmacological neuroprotection in neurodegenerative disease: assessing the preclinical data. J Neuropsychiatry Clin Neurosci. 2010;22(1):8-18.

13. Marksteiner J, Schmidt R. Treatment strategies in Alzheimer's disease with a focus on early pharmacological interventions. Drugs Aging. 2004; 21(7):415-426.

14. Massoud F, Gauthier S. Update on the pharmacological treatment of Alzheimer's disease. Curr Neuropharmacol. 2010;8(1):69-80.

15. Hogan DB. Progress update: pharmacological treatment of Alzheimer's disease. Neuropsychiatr Dis Treat. 2007;3(5):569-578.

16. Zec RF, Burkett NR. Non-pharmacological and pharmacological treatment of the cognitive and behavioral symptoms of Alzheimer disease. Neuro Rehabilitation. 2008;23(5):425-438.

17. Wimo A, Norlund A. Commentary on "health economics and the value of therapy in Alzheimer's disease." Cost-effectiveness studies. Alzheimers Dement. 2007;3(3):157-161.

18. Zhu CW, Sano M. Economic considerations in the management of Alzheimer's disease. Clin Interv Aging. 2006;1(2):143-154.

19. Peretti CS, Ferreri F, Blanchard F, et al. Normal and pathological aging of attention in presymptomatic Huntington's, Huntington's and Alzheimer's disease, and nondemented elderly subjects. Psychother Psychosom. 2008;77(3):139-146.

20. Solfrizzi V, Panza F, Torres F, et al. Selective attention skills in differentiating between Alzheimer's disease and normal aging. J Geriatr Psychiatry Neurol. 2002;15(2):99-109.

21. Daulatzai MA. Early stages of pathogenesis in memory impairment during normal senescence and Alzheimer's disease. J Alzheimers Dis. 2010;20(2):355-367.

22. Scheff SW, Price DA, Schmitt FA, Mufson EJ. Hippocampal synaptic loss in early Alzheimer's disease and mild cognitive impairment. Neurobiol Aging. 2006;27(10):1372-1384.

23. Dumont C, Voisin T, Nourhashemi F, Andrieu S, Koning M, Vellas B. Predictive factors for rapid loss on the mini-mental state examination in Alzheimer's disease. J Nutr Health Aging. 2005;9(3):163-167.

24. Chan DC, Kasper JD, Black BS, Rabins PV. Prevalence and correlates of behavioral and psychiatric symptoms in community-dwelling elders with dementia or mild cognitive impairment: the memory and medical care study. Int J Geriatr Psychiatry. 2003;18(2):174-182.

25. Garcia-Alberca JM, Lara JP, Berthier ML, et al. Can impairment in memory, language and executive functions predict neuropsychiatric symptoms in Alzheimer's disease (AD)? Findings from a cross-sectional study. Arch Gerontol Geriatr. 2011;52(3):264-269.

26. Vilalta-Franch J, Lopez-Pousa S, Turon-Estrada A, et al. Syndromic association of behavioral and psychological symptoms of dementia in Alzheimer disease and patient classification. Am J Geriatr Psychiatry. 2010;18(5):421-432.
27. Rinaldi S, Fontani V, Inventor; Rinaldi S, Fontani V, assignee Radioelectric Asymmetric Conveyer for therapeutic use. US patent $7,333,8592001$.

28. Rinaldi S, Fontani, V. Radioelectric Asymmetric Conveyer for therapeutic use. European Patent Office - World Intellectual Property Organization. October 11, 2006 2000(EP20010960475 20010706).

29. Mannu P, Rinaldi S, Fontani V, Castagna A. Long-term treatment of bipolar disorder with a radio-electric asymmetric conveyor. Neuropsychiatr Dis Treat. 2011;7(1):373-379.

30. Castagna A, Rinaldi S, Fontani V, Aravagli L, Mannu P, Margotti ML. Does osteoarthritis of the knee also have a psychogenic component? Psycho-emotional treatment with a radio-electric device vs intraarticular injection of sodium hyaluronate: an open-label, naturalistic study. Acupunct Electrother Res. 2010;35(1-2):1-16.

31. Collodel G, Moretti E, Fontani V, et al. Effect of emotional stress on sperm quality. Indian Journal of Medical Research. 2008;128(3): 254-261.

32. Mannu P, Rinaldi S, Fontani V, Castagna A, Lotti Margotti M. Radio electric treatment vs es-citalopram in the treatment of panic disorders associated with major depression: an open-label, naturalistic study. Acupunct Electrother Res. 2009;34:135-149.

33. Rinaldi S, Fontani V, Aravagli L, Margotti ML. Psychological and symptomatic stress-related disorders with radio-electric treatment: psychometric evaluation. Stress Health. 2010;26(5):350-358.

34. Rinaldi S, Fontani V, Aravagli L, Mannu P. Psychometric evaluation of a radio electric auricular treatment for stress related disorders: a double-blinded, placebo-controlled controlled pilot study. Health Qual Life Outcomes. 2010;8(1):31.

35. Rinaldi S, Fontani V, Moretti E, et al. A new approach on stress-related depression and anxiety: neuro-psycho-physical-optimization with radio electric asymmetric-conveyer. Indian Journal of Medical Research. 2010;132:189-194.

36. Soubelet A, Salthouse TA. Correlates of level and change in the minimental state examination. Psychol Assess. April 11, 2011.

37. Yoshino H, Sakurai T, Hasegawa K, Yokono K. Causes of decreased activity of daily life in elderly patients who need daily living care. Geriatr Gerontol Int. 2011;11(3):297-303.

38. Fujiwara Y, Chaves PH, Yoshida H, et al. Intellectual activity and likelihood of subsequently improving or maintaining instrumental activities of daily living functioning in community-dwelling older Japanese: a longitudinal study. Int J Geriatr Psychiatry. 2009;24(6): 547-555.

39. Zuidema SU, Buursema AL, Gerritsen MG, et al. Assessing neuropsychiatric symptoms in nursing home patients with dementia: reliability and reliable change index of the neuropsychiatric inventory and the cohen-mansfield agitation inventory. Int J Geriatr Psychiatry. 2011; 26(2):127-134.
Clinical Interventions in Aging

\section{Publish your work in this journal}

Clinical Interventions in Aging is an international, peer-reviewed journal focusing on evidence-based reports on the value or lack thereof of treatments intended to prevent or delay the onset of maladaptive correlates of aging in human beings. This journal is indexed on PubMed Central, MedLine, the American Chemical Society's 'Chemical Abstracts

\section{Dovepress}

Service' (CAS), Scopus and the Elsevier Bibliographic databases. The manuscript management system is completely online and includes a very quick and fair peer-review system, which is all easy to use. Visit http://www.dovepress.com/testimonials.php to read real quotes from published authors. 